\title{
EDITORIAL
}

\section{Who would I not give Ilb/llla inhibitors to during percutaneous coronary intervention?}

\section{J M McLenachan}

Glycoprotein Ilb/llla inhibitors should make the procedure of percutaneous coronary intervention safer for the patient. So should these agents be administered to every patient undergoing $\mathrm{PCl}$ ?

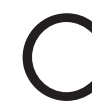
oronary artery stenting and platelet IIb/IIIa receptor antagonists confer complemen2 tary benefits on patients undergoing percutaneous coronary intervention (PCI). Glycoprotein $(\mathrm{Gp})$ IIb/IIIa inhibitors make the procedure safer by reducing periprocedural complications such as myocardial infarction, while stenting reduces restenosis rates and the need for further revascularisation procedures.

If Gp IIb/IIIa inhibitors make PCI safer, then should every PCI patient receive a Gp IIb/IIIa inhibitor? It is difficult to argue against this. Studies of Gp IIb/IIIa inhibitors with mandatory PCI have almost invariably shown benefit from active treatment; within these studies, multiple subgroup analyses have generally shown increased benefit in specific subgroups, particularly among diabetics patients and those with raised troponin concentrations. No subgroup analysis, however, has identified a group of patients that is harmed by adjunctive Gp IIb/IIIa inhibitor treatment during PCI.

\section{RISKS VERSUS BENEFITS}

The simplistic answer, therefore, to the question as to who should and who should not receive Gp IIb/IIIa inhibitors is that these agents should be given when the potential benefits to the individual patient outweigh the risks. This is likely to apply to the majority of patients undergoing PCI. However, there will be two groups of patients in whom the risks may outweigh the benefits. On the one hand, there will be patients in whom the risk of the procedure is so low that it is unlikely that the addition of a Gp IIb/IIIa inhibitor will confer any additional benefit. This group is likely to comprise non-diabetic patients with stable symptoms undergoing elective PCI in whom the procedure is uncomplicated and does not involve the use of multiple, or very long, stents. This is not a group of patients that has been extensively studied. Studies tend to concentrate on higher risk patient groups with more end points. Some data, however, are available on low risk patients. In the ESPRIT study, ${ }^{1}$ defined as a study of non-urgent coronary artery stenting, less than $50 \%$ of patients were, in the UK sense, "elective". While there was a clear benefit associated with eptifibatide treatment among those admitted
Great George Street, Leeds LSI 3EX, UK

carole.noble@leedsth.nhs.uk
.................... with an acute coronary syndrome, no such benefit was seen in the 794 patients with stable angina (six month death/myocardial infarction rate 6.9\% eptifibatide, $7.3 \%$ placebo, NS).

Non-randomised data sources also provide useful information. In the British Cardiovascular Intervention Society (BCIS) audit database for 2000, a subgroup of 3448 patients with complete hospital data underwent PCI for single vessel disease (excluding chronic total occlusions). ${ }^{2}$ The death rate was $0.12 \%$ (that is, four deaths during 3448 procedures). The National Confidential Enquiry into Perioperative Deaths (NCEPOD) report on percutaneous coronary angioplasty (PTCA), published in November 2000, showed similar results. ${ }^{3}$ Although there were 121 deaths in total, only 10 of these occurred during elective PTCA, equating to a mortality risk of less than $0.1 \%$. At such low levels of absolute risk, it seems unlikely that adjunctive Gp IIb/IIIa inhibitor treatment would confer a significant benefit; the low levels of absolute risk also mean that a study of this group would require very large numbers and is unlikely to be feasible.

The low risk associated with elective PCI has been recognised in the revised National Institute for Clinical Evidence (NICE) guidelines. In the original NICE guideline, published in September 2000, Gp IIb/IIIa inhibitors were recommended for all patients undergoing PCI, whether acute or elective. ${ }^{4}$ In the recently published revised guidance, ${ }^{5} \mathrm{Gp}$ IIb/IIIa inhibitors are recommended in many patient groups, including diabetic patients and acute coronary syndrome patients, but are no longer mandated in procedurally uncomplicated elective PCI where the risk of adverse events is low.

\section{HIGH RISK PATIENTS}

At the other end of the risk spectrum are patients at very high risk from the complications of the treatment. This is probably a small group since reported complication rates for Gp IIb/IIIa inhibitors are relatively low. In a meta-analysis of six randomised trials involving 31402 patients, major bleeding was more common in those treated with a Gp IIb/IIIa inhibitor $(2.4 \% \quad v$ $1.4 \%) .{ }^{6}$ Rates for intracranial haemorrhage, however, were low when compared to thrombolysis trials and were not significantly increased by Gp IIb/IIIa inhibitor treatment $(0.08 \%$ for active group, $0.05 \%$ for placebo, NS). Numbers for all strokes were higher but were still less than $1 \%$ and were not significantly higher among Gp IIb/IIIa inhibitor treated patients. Based on the published data, therefore, the risks associated with adjunctive Gp IIb/IIIa inhibitor treatment are low. 
In this edition of Heart, Brown presents some "real life" data on deaths associated with the use of glycoprotein IIb/IIIa inhibitors. ${ }^{7}$ This is not a randomised study but is a review of all those deaths reported to the US Food and Drug Administaration between November 1997 and December 2000 in which treatment with this class of drug was implicated. Of the 450 deaths reported, $44 \%$ were considered to be "definitely or probably" related to the use of Gp IIb/IIIa inhibitors. A disadvantage of the study is that the rate of major complications cannot be calculated since neither the exact number of PCI procedures, nor the number of patients being treated with IIb/ IIIa inhibitors during the study period, is known. The author points out, however, that those who died were generally older, and more likely to be female, than the patients included in the large scale randomised clinical trials of these agents. He questions whether we can apply the results of these trials to our everyday clinical practice where patients are often older, and sicker, than those patients enrolled in randomised studies.

The concept that clinical trial populations are relatively low risk, and not representative of everyday practice, is not new. In the recently published GUSTO V trial of standard dose reteplase versus half dose reteplase and full dose abciximab for the treatment of patients with ST segment elevation myocardial infarction, the 30 day mortality was $5.6 \%$ in the study group and $5.9 \%$ in the control group. ${ }^{8}$ In a recent observational study in Yorkshire, which enrolled "all comers" with myocardial infarction, the 30 day mortality was $24 \%{ }^{9}$ This four-fold difference in mortality is attributable to multiple differences between the two populations; the "all comers" included in observational studies tend to be older, to present later, to have a higher prevalence of previous cardiac events, and to have more co-morbidity than those patients enrolled into clinical trials.

The concept of age specific differences in the efficacy, and the complications, of different treatments is also not new. There has been considerable recent controversy around whether the results of thrombolysis trials, conducted mainly in younger patients presenting with a first myocardial infarction, can be applied to elderly patients. ${ }^{10}$ In all of these situations, the clinician has to balance the likely benefits and possible risks to the individual patient. This is not easy. The sickest patients, who often have most to gain from a given treatment, are also at highest risk of major, and possibly fatal, complications. This dilemma regularly confronts the interventionist when undertaking primary PCI or salvage PCI for failed thrombolysis or shock. In the UK, primary PCI for myocardial infarction is not a routine treatment. Instead, primary PCI is usually reserved for those patients who are ineligible for thrombolysis. The very factor that makes the patient unsuitable for thrombolysis (active bleeding, recent gastrointestinal of genitourinary bleeding, recent cerebrovascular accident, recent major trauma, etc) is also a relative contraindication to Gp IIb/IIIa inhibitor treatment. In salvage and shock patients, the bleeding risks associated with Gp IIb/IIIa inhibitor treatment are greatly increased because the patient has already received one or more thrombolytic or fibrinolytic drugs. In each case, the interventionist has to balance the potential beneficial effects with the bleeding risk. In most high risk PCI patients, the interventionist will usually wish to give a Gp IIb/IIIa inhibitor.

\section{ELDERLY PATIENTS}

GUSTO V provides some data both on the elderly and on the interaction between abciximab and fibrinolytics in the elderly. ${ }^{8}$ Of the 8328 patients in the GUSTO V acute myocardial infarction trial who received abciximab plus reteplase, 2135 $(26 \%)$ were aged 70 years and over. The incidence of moderate and severe gastrointestinal bleeding increased with age, particularly above the age of 75 . Patients above the age of 75 were the only age group with a trend towards increased intracranial haemorrhage in the reteplase plus abciximab arm compared to reteplase alone. However, there was no statistically or clinically relevant difference in the composite of death and non-fatal disabling stroke in patients over 75 years of age; furthermore, these patients appeared to benefit most from the combination treatment in terms of reduction of reinfarction.

Limited "real world" data are available from postmarketing surveillance. By December 2001, an estimated 1729329 patients had received abciximab (Reopro post marketing database, personal communication). Analysis of the postmarketing database suggested that the rates of major complications were consistent with the published trials; as in the trials, the risk of haemorrhagic complications was associated with the co-administration of plasminogen activators, fibrinolytic agents, and dextran-containing products.

In summary, Gp IIb/IIIa inhibitors should be given to the majority of patients undergoing PCI. Patients whom I would not treat with a Gp IIb/IIIa inhibitor fall into two groups: the $10-20 \%$ of PCI patients who are at very low risk (elective procedure, non-diabetic, single stent, procedurally uncomplicated, etc) and the very small number of ill patients with a life threatening condition likely to be exacerbated by IIb/IIIa inhibition (active bleeding, recent cerebrovascular accident, etc). As ever, guidelines give guidance but the clinician still has to balance the risks and possible benefits for the individual patient.

\section{REFERENCES}

1 O'Shea JC, Hafley GE, Greenberg S, et al. Platelet glycoprotein Ilb/Illa integrin blockade with eptifibatide in coronary stent intervention. The ESPRIT trial: a randomized controlled trial. JAMA 2001:285:2468-73.

2 British Cardiovascular Intervention Society. UK PCl audit data 2000 URL: http://www.bcis.org.uk/

3 NCEPOD. Percutaneous transluminal coronary angioplasty. A report of the National Confidential Enquiry into Perioperative Deaths. London: Department of Health, November 2000.

4 National Institute for Clinical Excellence. Guidance on the use of glycoprotein Ilb/Illa inhibitors in the treatment of acute coronary syndromes. NICE technology appraisal guidance No. 12. London: NICE, 2000.

5 National Institute for Clinical Excellence. Guidance on the use of glycoprotein Ilb/Illa inhibitors in the treatment of acute coronary syndromes. NICE technology appraisal guidance No. 47. London: NICE, 2002.

6 Boersma E, Harrington RA, Moliterno DJ, et al. Platelet glycoprotein $\mathrm{Ilb} / \mathrm{llla}$ inhibitors in acute coronary syndromes: a metanalysis of all major randomised clinical trials. Lancet 2002;359:189-98.

7 Brown DL. Deaths associated with platelet glyoprotein Ilb/Illa inhibitor treatment. Heart 2003;89:535-7.

8 The GUSTO V Investigators. Reperfusion therapy for acute myocardial infarction with fibrinolytic therapy or combination reduced fibrinolytic therapy and platelet glycoprotein Ilb/Illa inhibition: the GUSTO V randomised trial. Lancet 2001;357:1905-14.

9 Dorsch MF, Lawrance RA, Sapsford R, et al. A simple benchmark for evaluating quality of care of patients admitted following acute myocardial infarction. Heart 2001;86:150-4.

10 Thiemann DR, Coresh J, Schulman SP, et al. Lack of benefit for intravenous thrombolysis in patients with myocardial infarction who are older than 75 years. Circulation 2000;101:2239-46. 